\title{
ON THE JETS ASSOCIATED WITH GALACTIC SUPERLUMINAL SOURCES
}

\author{
Amir LeVinson ${ }^{1}$ AND Roger Blandford \\ Theoretical Astrophysics 130-33, California Institute of Technology, Pasadena, CA 91125 \\ Received 1995 July 6; accepted 1995 October 19
}

\begin{abstract}
Recent observations of GRS 1915+105 and GRO J1655+40 reveal superluminal motions in Galactic sources. This Letter examines the physical conditions within these Galactic sources, their interaction with their environment, and their possible formation, and contrasts them with their extragalactic counterparts. In particular, $e^{+}-e^{-}$and $e-p$ jets are contrasted; constraints on particle acceleration in the jets are imposed using X-ray and radio observations; and the $\gamma$-ray flux from $e^{+}-e^{-}$jets expected at EGRET energies and the flux in infrared lines from an $e-p$ jet are estimated. It is also suggested that these sources may exhibit low-frequency radio lobes extending up to several hundred parsecs in size, strong, soft X-ray absorption during the birth of the radio components, and emission-line strengths anticorrelated with the X-ray flux. The implications for other X-ray transients are briefly discussed.
\end{abstract}

Subject headings: galaxies: jets — radio continuum: stars — stars: individual (GRS 1915+105, GRO J1655+40)

\section{INTRODUCTION}

There is now direct evidence for superluminal motion in the radio images of two strong Galactic X-ray transient sources, GRS 1915+105 and GRO J1655+40 (Mirabel \& Rodriguez 1994, hereafter MR94; Tingay et al. 1995; Hjellming \& Rupen 1995, hereafter HR95). These motions are probably associated with relativistic jets emanating from a black hole in an X-ray binary. Several other transient radio sources associated with soft X-ray novae may also involve collimated jets (HR95). In this Letter we use existing radio, optical, and X-ray observations to place constraints on the physical conditions within these radio-emitting X-ray transients, contrasting them with their extragalactic counterparts. We analyze the jets in some generality, speculate upon how they may be collimated, and illustrate these ideas using GRS $1915+105$ and GRO $\mathrm{J} 1655+40$.

\section{RELATIVISTIC JETS}

\subsection{Synchrotron and Inverse-Compton Emission}

Consider a jet formed at $r \equiv 10^{x} r_{\mathrm{X}} \mathrm{cm} \sim 10^{6} \mathrm{~cm}$ near a black hole in an X-ray binary of orbital radius $a \sim 10^{12} \mathrm{~cm}$. From the resolved radio synchrotron emission, we define a fiducial equipartition field strength $B^{*} \sim 4\left(T_{B 6} / \phi_{-1} r_{16}\right)^{2 / 7} \nu_{9}^{5 / 7} \mathrm{mG}$, where the brightness temperature $T_{B}=10^{6} T_{B 6} \mathrm{~K}$ is evaluated at the frequency $\nu=\nu_{9} \mathrm{GHz}, \phi=0.1 \phi_{-1} \mathrm{rad}$ is the opening angle, and the radio spectral index is $\alpha_{R} \sim 0.5$ (cf. Rybicki \& Lightman 1979). Let the jet Lorentz factor be $\Gamma$, and let its velocity make an angle $\theta$ with the line of sight. The jet power associated with the emitting electrons and the electromagnetic field is then given by

$$
L_{\text {jet }} \gtrsim \frac{c}{2}\left(\frac{\Gamma B^{*} \phi r}{\delta_{o}^{5 / 7}}\right)^{2} \sim 10^{34} \Gamma^{2} \delta_{o}^{-10 / 7}\left(B_{-3}^{*}\right)^{2} \phi_{-1}^{2} r_{16}^{2} \operatorname{ergs~s}^{-1},
$$

where $\delta_{o}=[\Gamma(1-\beta \cos \theta)]^{-1}$ and rough equality occurs at equipartition.

When the speed of advance of the jet's head $V_{h}$ is supersonic, strong shocks may be formed at the outer lobes, as in

${ }^{1}$ Postal address: CRSR, Space Sciences Building, Cornell University, Ithaca, NY 14853. extragalactic FR II sources, and can give rise to hot spots and low-frequency lobes. When $V_{h}$ is subsonic, there may be a FR I type source (e.g., Begelman, Blandford, \& Rees 1984).

To estimate the maximum radio power emitted by the lobes, we assume that the lobes subtend $\sim 1 \mathrm{sr}$ so that their pressure is $\sim L_{j}^{2 / 3} \rho^{1 / 3} r^{-4 / 3} \sim 3 \times 10^{-7} L_{j 38}^{2 / 3} n^{1 / 3} r_{18}^{-4 / 3}$ dyn $\mathrm{cm}^{-2}$. The lobe brightness temperature at a fiducial frequency $\sim 5 \mathrm{GHz}$ then satisfies $T_{B 5} \lesssim 4 \times 10^{5} L_{j 38}^{7 / 6} n^{1 / 2} r_{18}^{-4 / 3} \mathrm{~K}$. These lobes may be detectable against the normal Galactic radio background.

Accelerated relativistic electrons can also radiate by inverseCompton scattering of accretion disk radiation. Let the energy density be dominated by photons of energy $E_{\mathrm{X}} \sim 1 \mathrm{keV}$ (in contrast to $\sim 10 \mathrm{eV}$ for extragalactic sources) and luminosity $L_{s}(r)$, and let the characteristic Doppler factor for transforming this radiation into the frame of the jet be $\delta_{j}(r)$ so that the associated photon energy in the jet frame is $E_{\mathrm{X}}^{\prime} \sim \delta_{j} E_{\mathrm{X}}$. Introduce a characteristic electron cooling energy in the jet frame by equating the radiative cooling time to the outflow timescale

$$
E_{\mathrm{ec}}^{\prime}(r) \sim 3\left(\frac{\Gamma}{\beta k_{j}}\right) L_{s 38}^{-1} r_{12} \mathrm{GeV},
$$

where $k_{j} \sim\left\langle\delta_{j}^{2}\right\rangle \sim \Gamma^{2} r_{7}^{-4}$ for direct illumination by the accretion disk and $k_{j} \sim \Gamma^{2} \tau$, if this radiation is scattered locally by free electrons in the surrounding medium with local Thomson depth $\tau$. Inverting equation (2), we can define a cooling radius $r_{c}\left(E_{e}^{\prime}\right)$, within which electrons of energy $E_{e}^{\prime}$ will cool. In order to accelerate an electron to an energy $\gtrsim E_{\text {ec }}^{\prime}$ requires that the particle acceleration occur impulsively on a timescale $t_{\text {acc }}^{\prime}<r /$ $\Gamma c$. The maximum $\gamma$-ray energy that can be scattered is then $\delta_{o}\left(r / \Gamma c t_{\mathrm{acc}}^{\prime}\right) E_{\mathrm{ec}}^{\prime}$. The scattered flux and spectrum depend upon the fraction of the jet kinetic energy that is transformed into relativistic particles in this manner. If a fraction $\eta$ of the jet power is emitted as $\gamma$-rays, the integrated $\gamma$-ray flux at Earth will be

$$
F_{\gamma} \sim 10^{-8} \eta \delta_{o}^{2} L_{j 38} D_{4}^{-2} \operatorname{ergs~s}^{-1} \mathrm{~cm}^{-2},
$$

where $D_{4}$ is the distance to the source in $10^{4}$ pc. Further features of the Compton scattering depend upon whether the jet is pair- or proton-dominated. 


\subsection{Positronic versus Protonic Jets}

If $\gamma$-rays are emitted at small enough radius, they will not be able to escape without creating electron-positron pairs. These, in turn, can produce lower energy $\gamma$-rays, and a cascade will develop that terminates when the $\gamma$-ray has a low enough energy to escape. The region from which $\gamma$-rays of a given energy can escape is known as the $\gamma$-sphere, and its radius is

$$
r_{\gamma}\left(E_{\gamma}\right) \simeq 3 \times 10^{7} k_{p p} L_{E 44}\left(m_{e}^{2} c^{4} / E_{\gamma}\right) \mathrm{cm},
$$

where $10^{44} L_{E 44} \mathrm{~s}^{-1}$ is the spectral luminosity of the central X-ray source, $k_{p p} \sim\langle 1-\cos \phi\rangle$ for radiation from an accretion disk that propagates at an angle $\phi$ to the jet, and $k_{p p} \sim \tau$ if the local scattered component dominates (cf. Blandford \& Levinson 1995). We can invert equation (4) to define the threshold energy $E_{\gamma \mathrm{th}}(r)$, which is the maximum energy of an escaping $\gamma$-ray from radius $r$.

Now, pairs will cool to subrelativistic energies for $r \lesssim r_{c}\left(m_{e} c^{2}\right)$ $\sim 3 \times 10^{8} k_{j} L_{s 38} / \Gamma \mathrm{cm}$. Their density will be limited by annihilation (Blandford \& Levinson 1995). We can also define an annihilation radius, $r_{\text {ann }}$, within which the density of annihilated pairs becomes smaller than that required to carry the jet power. Consequently, pair jets require the presence of some other carrier of energy and momentum. (This may be a problem for the model of Liang \& $\mathrm{Li}$ 1995.) In the absence of baryons, this is presumably electromagnetic. For subrelativistic pairs, $r_{\mathrm{ann}} \sim 3 \times 10^{9} L_{j 38} \Gamma^{-1}$ $\mathrm{cm}$ and is somewhat less if the pairs remain relativistic.

Another important difference between the Galactic and the extragalactic sources is that the former have much steeper $\mathrm{X}$-ray spectra, and consequently their $\gamma$-spheric radii increase more rapidly with increasing $\gamma$-ray energy. Furthermore, $r_{\text {ann }} \sim r_{\gamma}(1 \mathrm{GeV})$, instead of $\sim r_{\gamma}(1 \mathrm{MeV})$ as in the extragalactic case. This probably means that the $\gamma$-ray spectrum will be flatter in the $\mathrm{MeV}-\mathrm{GeV}$ range. Furthermore, the bulk Lorentz factors of the jets in the bright EGRET active galactic nucleus (AGN) sources are typically of order 10, much larger than those inferred for the jets in the Galactic sources. A plausible picture of Galactic $e^{ \pm}$jets, based on the above results, is as follows: some fraction of the accretion luminosity (or the spin energy of a rotating black hole) is extracted from the central source in the form of a collimated electromagnetic jet. During quiescent states, the jet is essentially invisible. However, when either the particle acceleration is sufficiently rapid or a reduced ambient radiation field renders the inverse-Compton radiation loss sufficiently ineffective, pairs can be injected to energies above $E_{\gamma}(r)$, and an intense pair cascade is initiated. At this radius, a transition to a particle-dominated flow occurs via the evolution of the cascade, which leads to $\gamma$-ray emission and the eventual formation of a superluminal radio feature. If the cascade is initiated within the annihilation radius, the mildly relativistic pairs will be annihilated, and the radio spectrum will exhibit a low-energy cutoff.

If the jet is accelerated and collimated close to the black hole as an e-p plasma, perhaps through the agency of radiation pressure, and particle acceleration is inefficient above $\gtrsim E_{\gamma \mathrm{ht}}(r) / \Gamma$, then pairs are not created, and the minimum jet power is larger than that given by equation (1) by a factor $\sim\left[m_{p} / \gamma_{\min } \ln \left(\gamma_{\max } /\right.\right.$ $\left.\left.\gamma_{\min }\right) m_{e}\right]^{4 / 7}$, where the electron distribution function is supposed to extend from $\gamma_{\min }$ to $\gamma_{\max }$. Typically, this factor is $\sim 3-30$. (Alternatively, a pair jet may form as described above and plasma from the surrounding wind may be entrained.)

One possible diagnostic of $e-p$ jets is the presence of Doppler-shifted spectral lines, such as $\mathrm{H} \alpha$, as seen in SS 433 .
Because of the relativistic motion of the jet, the line will be Doppler shifted by the approaching and receding Doppler factors $\delta_{o}$. Following Begelman et al. (1980) and Davidson \& McCray (1980), we suppose that the gas in the line-emitting region is clumped, and we denote by $\varepsilon$ the volume-filling factor of the dense blobs comprising the line-emitting beam and by $10^{15} R_{15} \mathrm{~cm}$ the beam's length. The $\mathrm{H} \alpha$ emissivity should lie in the range between $10^{-24.6}$ and $10^{-23} \mathrm{ergs} \mathrm{cm}^{3} \mathrm{~s}^{-1}$ at $\sim 10^{4} \mathrm{~K}$ (Davidson \& McCray 1980), depending on the density in the emitting blobs. Let us adopt the value $10^{-23.5} \mathrm{ergs} \mathrm{cm}^{3} \mathrm{~s}^{-1}$. We then obtain for the emitted flux

$$
F_{\mathrm{H} \alpha} \simeq 10^{-23} \delta^{2}\left(L_{j 38} / D_{4}\right)^{2}\left(\varepsilon R_{15} \phi^{2} \Gamma^{4}\right)^{-1} \mathrm{ergs} \mathrm{cm}^{-2} \mathrm{~s}^{-1},
$$

where it has been assumed that the average density of the hot phase is $\varepsilon n_{\text {cold }}$. The cold blobs should be confined by the pressure of the hot phase in the jet or, alternatively, by the magnetic fields.

\section{JET FORMATION AND CONFINEMENT}

The Galactic superluminal sources further demonstrate that relativistic jet formation can operate on a stellar as well as a galactic scale. Presumably, the common feature is the presence of an accretion disk orbiting in a relativistically deep potential well. In order to explore how this might occur in a $(M \sim$ 3-10 $M_{\odot}$ ) binary X-ray source, we suppose that the jet is collimated by a wind emanating from the disk surface over a range of radii from $\lesssim 10^{7}$ to $\gtrsim 10^{11} \mathrm{~cm}$ with speed $V_{W}=$ $1000 V_{W 8} \mathrm{~km} \mathrm{~s}^{-1}$ declining with cylindrical radius. As the jet propagates away from its source, there will be radial transport of linear momentum, which will flatten the velocity profile. If most of the momentum derives from large disk radius, then the asymptotic jet speed will be $V_{W 8} \sim 1-10$.

It has long been argued in the case of AGNs and protostellar jets that a hydromagnetic wind is a more plausible collimator than a purely hydrodynamic wind because when the field is primarily toroidal, the transverse force density is $-r_{\perp}^{-2} d\left(r_{\perp}^{2} P_{\text {mag }}\right) / d r_{\perp}$ (as opposed to $-d P_{\text {gas }} / d r_{\perp}$ for gas pressure), which allows a large jet pressure to be confined by a much smaller external pressure. In addition, in the expanding wind, $P_{\text {mag }}$ declines less rapidly than $P_{\text {gas }}$, which implies that magnetic collimation is likely to become relatively more important. We adopt the magnetic collimation hypothesis, although much of what follows is more general.

For $r \ll a$, magnetic confinement of the jet can be relatively effective with each nested magnetic surface confining the interior flow, until ultimately the inertia of the wind from the outer disk prevents transverse expansion. However, this magnetic focusing cannot provide much pressure amplification after the jet has propagated out to a radius comparable with the outer radius of the disk. At this point, either the jet itself must have sufficient internal density to be effectively free and travel hypersonically with Mach number $\gtrsim \phi^{-1}$ or it must be confined by the inertia of the surrounding wind. The former possibility may be relevant for $e-p$ jets. However, we suspect that pair jets require external confinement at this radius. The foregoing considerations suggest that the jet itself exerts a transverse pressure of

$$
P \sim 3 \times 10^{4} L_{j 38} \Gamma^{-2} \beta^{-1} r_{12}^{-2} \phi_{-1}^{-2} \text { dyn } \mathrm{cm}^{-2},
$$

which will cause the surrounding, slower wind to expand with a speed $\sim\left(P / \rho w_{j}\right)^{1 / 2} \lesssim V_{W_{j}} \phi$, where $W j$ denotes values of the wind density and speed averaged within a few jet widths. The 
minimum wind discharge for ultimate inertial confinement at radius $r \sim a$ is then given by

$$
\dot{M}_{W j} \gtrsim 10^{-4} L_{j 38} \Gamma^{-2} \beta^{-1} \phi_{-1}^{-2} V_{W j 8}^{-1} M_{\odot} \mathrm{yr}^{-1},
$$

and the associated wind power is $\gtrsim 10^{38} L_{j 38} \Gamma^{-2} \beta^{-1} \phi_{-1}^{-2} V_{W j 8}$ ergs $\mathrm{s}^{-1}$. (In making this estimate, we have supposed that most of the discharge is confined to a polar wind with transverse scale $\sim 3$ jet radii. If the wind fills a larger solid angle, the discharge and power must correspondingly be increased.) This wind will propagate well beyond the observed radio sources before terminating through a strong shock when its momentum flux balances the ambient interstellar pressure.

If we measure the wind discharge as $\sim 10^{-6} \dot{M}_{W j-6} M_{\odot} \mathrm{yr}^{-1}$, its Thomson optical depth is likely to be $\tau_{\mathrm{T}}(r) \sim 0.1 \dot{M}_{W j-6} V_{W 8}^{-1} r_{12}^{-1}$, for $r_{12} \gtrsim 0.1$. For $r_{12} \lesssim 0$.1, we emphasize that the optical depth need not be much greater than this value because of the efficiency of magnetic confinement. However, the wind that we postulate is likely to extinguish any soft X-ray flux and variations if it is strong enough to collimate the jets.

The thermal state of the wind depends upon the photoionizing flux. The ionization parameter is $U \sim 0.1 L_{\mathrm{UV} 36} V_{W 8} / \dot{M}_{W j-6}$. For $0.1 \lesssim \mathrm{U} \lesssim 10$, a two-phase medium is possible with hot Compton-heated gas at a temperature $T \sim 10^{7}-10^{8} \mathrm{~K}$ coexisting with line-emitting gas at a temperature $T \sim 10^{4} \mathrm{~K}$. (At the density envisaged, the thermal equilibration time turns out to be short compared with the outflow time.) Now suppose that the mass accretion rate increases as a consequence of some disk instability. The ultraviolet and X-ray emission will increase as a consequence of enhanced dissipation at the inner disk. This will heat the gas so that the pressure is largely thermal as opposed to largely magnetic. We suggest that this prevents effective magnetic collimation, and consequently a jet does not form. When the disk accretion rate falls, the ionization parameter falls, and the gas in the wind cools so that it becomes magnetically dominated. This allows a collimating hydromagnetic wind to form. If there is also a central source of relativistic plasma or electromagnetic energy, perhaps derived from the spin of a central black hole, then this can form the radio-emitting core of the radio jet (cf. Meier 1995). This wind is also likely to be a source of optical and ultraviolet emission lines, and our model predicts that the fraction of the bolometric flux reprocessed in the form of emission lines should be anticorrelated with the X-ray flux.

\section{INTERPRETATION OF GRS 1915+105 AND GRO J1655-40}

\subsection{GRS $1915+105$}

This source is at a distance of $D_{4} \sim 1.25$ (MR94) and exhibits X-ray luminosity of a few times $10^{38} \mathrm{ergs} \mathrm{s}^{-1}$ (Harmon et al. 1994). It has been observed by the VLA at 5 and $10 \mathrm{GHz}$. Following MR94, we assume, for simplicity, that the pattern speed and the flow speed are equal (c.f. Bodo \& Ghisellini 1995). The inferred speed and angle to the line of sight of the ejecta are then $\beta \sim 0.92$ and $\theta=70^{\circ} \pm 2^{\circ}$ (MR94), corresponding to $\delta_{o} \simeq 0.57$. (This measurement allows us to predict the wavelengths of, for example, possible $\mathrm{H} \alpha$ lines, namely $\sim 1.15,2.15 \mu \mathrm{m}$.) The radio features appear to move away at a constant speed out to a distance of at least $0.1 \mathrm{pc}$ from the central source (MR94).

On 1994 March 24, the measured flux was $~ 0.7$ Jy. Even though the source was not resolved at that time, the inferred distance from the putative core was $\sim 0 . " 08$, and the blob size was $\sim 0$."06, corresponding to a linear size of $\sim 10^{16} \mathrm{~cm}$. We estimate
$P_{\min } \simeq 1.8 \times 10^{-5} \mathrm{dyn}^{-2}$, and $B^{*} \simeq 2 \times 10^{-2} \mathrm{G}$, at a distance of $\sim 10^{16} \mathrm{~cm}$ from the central source. Equation (1) then gives $L_{j 38} \gtrsim 2$ for an $e^{ \pm}$jet and $\sim 4$ times that for an $e-p$ jet. The annihilation radius $r_{\text {ann }} \gtrsim 2.4 \times 10^{9} \mathrm{~cm}$. Taking $\kappa_{p p} L_{s 38}=10^{-2}$ yields $r_{\gamma} \simeq 3 \times 10^{8}\left(E_{\gamma} / 1 \mathrm{GeV}\right)^{0.5} \mathrm{~cm}$. Since $r_{\gamma}(1 \mathrm{GeV})<r_{\mathrm{ann}}$ a low-frequency cutoff of the radio spectrum from the jet may be expected. The acceleration time required for a formation of a particle-loaded blob is $\lesssim 10^{-3}(r / c)$. From equation (3) it follows that $F_{\gamma} \gtrsim 3 \times 10^{-9} \eta$ ergs $\mathrm{cm}^{-2} \mathrm{~s}^{-1}$. If the radiative efficiency $\gtrsim 0.1, \gamma$-ray outbursts from a pair jet might be detectable. Alternatively, for a protonic jet, we estimate that $F_{\mathrm{H} \alpha} \lesssim 10^{-14}$ ergs $\mathrm{s}^{-1} \mathrm{~cm}^{-2}$, which might be detectable.

Recently, Rodriguez \& Mirabel (1995) have reported the discovery of two $20 \mathrm{~cm}$ radio sources $(80,40 \mathrm{mJy})$, coincident with two $0.5 \mathrm{kJy}, 100 \mu \mathrm{m} I R A S$ sources both $17^{\prime}(\sim 60 \mathrm{pc})$ from GRS $1915+105$. The sources are colinear with position angle close to that of the radio ejections. The infrared power is $\sim 3 \times 10^{38}$ ergs s $^{-1}$, which suggests a high radiative efficiency. The individual components are both elongated along the same position angle. Rodriguez \& Mirabel suggest that these two sources are $\mathrm{O}$ stars surrounded by $\mathrm{H}$ II regions.

Alternatively, the hot spots might be synchrotron emission from the ends of the jets. If so, then we can estimate the jet power by assuming that the size of the hot spots is $\sim l$ pc and the shocked jet fluid flows through this region with mildly relativistic speed. We find that $L_{j} \sim 5 \times 10^{39} k^{3 / 7} l^{-2 / 7}$ ergs s ${ }^{-1}$, where $k \gtrsim 1$ is the ratio of the total pressure to the relativistic particle pressure. For $l \sim 3, k \sim 1$, this is within an order of magnitude of the jet power inferred on the basis of the observed jets. The inferred pressure is $\sim 10^{-8}$ dyn $\mathrm{cm}^{-2}$. However, the radio flux density is much smaller than the 100 $\mu \mathrm{m}$ flux density, in contrast to other optically thin synchrotron sources. One possible explanation is free-free absorption, which can occur if there is ionized gas present with density $\sim 3 \times 10^{3} \mathrm{~cm}^{-3}, T \sim 10^{4} \mathrm{~K}$, in rough equipartition with the relativistic electrons and magnetic field. Perhaps this is cool gas behind a radiative bow shock surrounding the advancing hot spot. If so, the characteristic age of the radio source will be $\sim 3 \times 10^{5} \mathrm{yr}$, just compatible with the estimated mass-loss rate.

\subsection{GRO J1655-40}

For this source, we adopt the parameters inferred by HR95, namely, $D_{4}=0.31$ (this distance is in a very good agreement with the distance inferred by Bailyn et al. 1995, based on interstellar absorption), $\beta=0.92$, and $\delta_{o} \simeq 0.46$. (The predicted $\mathrm{H} \alpha$ wavelengths are $1.4,1.8 \mu \mathrm{m}$.) The lowest frequency observed with the VLA was $\nu_{10}=0.15$. The light curves indicate a peak flux of $5.5 \mathrm{Jy}$ at this frequency 6 days after the beginning of the observations, implying $l<10^{16} \mathrm{~cm}$, and $T_{B 5} \sim 10^{7} \mathrm{~K}$. During the high X-ray state, Harmon et al. (1995) derived a $20-100 \mathrm{keV}$ luminosity of $\sim 10^{37} \mathrm{ergs} \mathrm{s}^{-1}$ and photon spectral index of about -3 , extending to at least $700 \mathrm{keV}$ (Kaaret et al. 1995). If we extrapolate the spectrum down to $1 \mathrm{keV}$, we estimate $L_{s 38} \lesssim 1$ during high states. However, the $\mathrm{X}$-ray luminosity was in fact smaller during the peak of the radio flux, as discussed below. Kaaret et al. (1995) also report that the power spectrum of soft X-ray fluctuations exhibits no features and little power above $0.1 \mathrm{~Hz}$, which is consistent with a wind being optically thick to Thomson scattering out to a radius $\sim 10^{11} \mathrm{~cm}$.

For illustration we shall assume $\kappa_{p p} L_{s 38}=10^{-3}$. Adopting these parameters we obtain $P_{\min } \simeq 5 \times 10^{-6}$ dyn $\mathrm{cm}^{-2}$, 
$B^{*} \simeq 10^{-2} \quad \mathrm{G}, \quad L_{j 38}>0.6, \quad r_{\mathrm{ann}}>7 \times 10^{8} \mathrm{~cm}, \quad$ and $\quad r_{\gamma} \sim$ $10^{7}\left(E_{\gamma} / 10^{3}\right)^{0.5} \mathrm{~cm}$, for an $e^{ \pm}$jet. For an $e-p$ jet, $L_{j 38} \gtrsim 3$. The $\mathrm{H} \alpha$ flux obtained from equation (5) is roughly the same as that obtained for GRS 1915+105.

Bailyn \& Orosz (1995) have measured a spectroscopic orbital period of 2.6 days (similar to the period inferred by HR95 and the proposed eclipse period of Bailyn et al. 1995) and a mass function $f_{1}=3.35 \pm 0.14 M_{\odot}$, implying that the compact object is a black hole with a mass $\sim 5.3 M_{\odot}$ and $a \sim 1 \times 10^{12} \mathrm{~cm}$. They have also reported observations of a hard optical continuum with spectral index $d \ln F_{v} / d \ln \nu \sim 0.3$ (similar to that expected from a classic accretion disk) as well as an emission-line spectrum exhibiting Balmer lines and $\mathrm{He} \mathrm{I}$ and a $\mathrm{F} / \mathrm{G}$ stellar spectrum. The presence of eclipses verifies that the inclination $i \sim 85^{\circ}$. After correcting for reddening, the optical luminosity is $\sim 0.01$ times the X-ray power and is comparable with that expected from a wind of the strength that we have had to posit to account for the radio jet collimation. The line widths are also compatible with those expected from a wind with $V_{W 8} \sim 1$. The hard X-rays are not subject to strong eclipse (Kaaret et al. 1995). All of this is consistent with the model inferred on the basis of the radio data. The secondary star is a $\sim 1 M_{\odot}$ dwarf of radius $\lesssim 10^{11} \mathrm{~cm}$ lying well within its Roche lobe (of radius $\sim 3 \times 10^{11} \mathrm{~cm}$ ). The optical and hard X-ray emission emerges from a fluctuating Thomson photosphere of similar size, associated with the disk wind. This accounts for intermittent, partial eclipses. One outstanding question raised by this model is the mode of mass transfer. We note that the power incident upon the secondary $\left(\sim 3 \times 10^{35} \mathrm{ergs} \mathrm{s}^{-1}\right)$ is marginally sufficient to transfer mass at the deduced rate of $\sim 10^{-6} M_{\odot} \mathrm{yr}^{-1}$ through the $L_{1}$ point assuming total efficiency.

\section{FUTURE OBSERVATIONAL TESTS}

The existence of collimated relativistic outflows in these two Galactic superluminal sources is a strong motivation for a search for other examples, particularly in known X-ray transients (HR95). (Observations in the week following X-ray outbursts are particularly relevant in view of the reported behavior in GR0 1655-40.) The two Galactic $\gamma$-ray sources, 1E 1740.7-2942 and GRS 1758-258 (Mirabel 1994; Chen, Gehrels, \& Leventhal 1994), show many similarities to the two sources considered above (radio jets, hard X-rays, upper limits on the masses of the stellar companions), as well as some differences (detected $\gamma$-rays, correlation between the radio and X-ray fluxes). It is also of interest to reexamine Cyg X-3 from which Strom, van Paradijs, \& van der Klis (1988) report mildly relativistically moving radio components.

The counterparts of the giant radio lobes by which the extragalactic counterparts of these sources were first recognized (or alternatively of W50) should also be sought. This search should be most profitably carried out at low frequency, and, as in the extragalactic case, the radio source sizes may be very large $\left(\gtrsim 10^{\prime}\right)$, depending upon the history and local gas density. As extragalactic observations also emphasize, there are possible strong selection effects, and GRS $1915+105$ and GRO 1655-40 may be modest Lorentz factor $(\Gamma \sim 2)$ examples of a much larger class, most of which contain ultrarelativistic jets $(\Gamma \gtrsim 10)$ rendered invisible by beaming away from us. Perhaps 1E 1700-2942 and GRS 1758-258 belong to this class. In addition, perhaps sources like A0620-00 are counterparts of radio-quiet quasars.

Also drawing upon the extragalactic analogy, a search for $\sim 1 \mathrm{GeV} \gamma$-rays using EGRET is well motivated, and a successful detection would strengthen the case for $e^{+}-e^{-}$jets. Conversely, the detection of optical or infrared Dopplershifted emission lines would strengthen the association with SS 433 and argue for an $e-p$ jet.

Given the large inclination derived from the radio source kinematics $\left(i \sim 84^{\circ}\right.$; HR95), a measurement could then be translated into an estimate of the size of the Roche lobe of the companion star. Understanding the size of the orbit and consequently of the accretion disk should also help define the physical conditions in the bipolar wind that we have invoked to account for the jet collimation. Further constraints on the discharge in the wind can come from observing the soft X-rays with ROSAT at energies $\lesssim 1 \mathrm{keV}$ during the phases when the radio components are being formed. If the outflow is as dense as we propose, then the soft X-rays should be efficiently absorbed. A quite separate bound on the wind density may be derivable by seeking rapid variability in hard X-rays using $A S C A$ during high states. Observation of rapid variability on timescale $t_{\mathrm{var}}$ would imply that any wind would be optically thin to Thomson scattering beyond a radius $r \sim c t_{\mathrm{var}}$. If, somewhat unexpectedly, the compact object in either source is a neutron star with a measurable spin period, then this test will be much stronger. In addition, we have suggested that the relative strength of the emission lines from this wind should be anticorrelated with the X-ray flux.

However, perhaps the most fundamental understanding as to the nature of these sources will come from analyzing the kinematics of the radio components to see if there are genuine periodicities in either the timing of the outbursts or, as tentatively supported by the observations of HR95, the component angular velocities projected on the sky. It will be especially interesting to learn if, on these grounds, the Galactic superluminal sources are more affiliated with extragalactic jets or SS 433.

We acknowledge helpful conversations with Charles Bailyn, Bob Hjellming, Dave Meir, Felix Mirabel, Rashid Sunyaev, and participants in the ITP workshop on $\gamma$-ray observations. Support under NASA grant NAG 5-2757 and NSF contract PHY 94-07194 is gratefully acknowledged.

\section{REFERENCES}

Bailyn, C. D., et al. 1995, Nature, 374, 701

Bailyn, C. D., \& Orosz, J. 1995, IAU Circ., No. 6173

Begelman, M., Blandford, R. D., \& Rees, M. 1984, Rev. Mod. Phys., 56, 255

Begelman, M., et al. 1980, ApJ, 238, 722

Blandford, R. D., \& Levinson, A. 1995, ApJ, 441, 79

Bodo, G., \& Ghisellini, G. 1995, ApJ, 441, L69

Chen, W., Gehrels, N., \& Leventhal, M. 1994, ApJ, 426, 586

Davidson, K., \& McCray, R. 1980, ApJ, 241, 1082

Harmon, A., et al. 1994, in Proc. 2d Compton Symp., ed. C. E. Fichtel, N. Gehrels, \& J. P. Norris (New York: AIP), 210

. 1995, Nature, 374, 703

Hjellming, R. M., \& Rupen, M. P. 1995, Nature, 375, 464 (HR)
Kaaret, P., et al. 1995, paper presented at Inst. for Theoretical Physics, Workshop on $\gamma$-Ray Astrophysics

Liang, E., \& Li, H. 1995, A\&A, 298, L45

Meier, D. 1995, preprint

Mirabel, I. F. 1994, ApJS, 92, 369

Mirabel, I. F., \& Rodriguez, L. F. 1994, Nature, 371, 46 (MR)

Rodriguez, L. F., \& Mirabel, I. F. 1995, in Superluminal Radio Sources, ed. M. Cohen \& K. I. Kellerman (Washington: National Acad. of Sciences), in press

Rybicki, G., \& Lightman, A. P. 1979, Radiation Processes in Plasma (New York: Wiley)

Strom, R. G., van Paradijs, J., \& van der Klis, M. 1988, Nature, 337, 234

Tingay, S. J., et al. 1995, Nature, 374, 141 